\title{
Novel Technique for Comprehensive Noise Identification and Cancellation in GSM Signal
}

\author{
Rekha $\mathbf{N}^{1}$, Fathima Jabeen ${ }^{2}$ \\ ${ }^{1}$ Department of Electronics \& Communication Engineering, K.S. Institute of Technology, Bangalore, India \\ ${ }^{2}$ Islamiah Institute of Technology, Bangalore, India
}

\begin{tabular}{l} 
Article Info \\
\hline Article history: \\
Received Jul 13, 2017 \\
Revised Jan 1, 2018 \\
Accepted Jan 8, 2018 \\
\hline
\end{tabular}

Keyword:

Allocated power

GSM signal

Interference

Transient signal

\begin{abstract}
Presence of noise significantly degrades the contents being transmitted over GSM channel. With the evolution of next generation of communication system, the challenges in noise cancellation in voice along with data transmission are not being addressed effectively by existing filters. Therefore, the proposed system offers a mechanism where emphasis is laid on identification of superior and inferior forms of GSM transient signal followed by cancellation of its noise level. Designed using analytical methodology, the proposed system harness the potential of probability theory to perform a modeling that associates allocated power of the transmitting device with the level of noise. The outcome of the study is found to offer a comprehensive identification of different forms of noise and can precisely determine the level of superior and inferior quality of signal. The outcome significantly assists in designing an accurate filter for noise cancellation in GSM signal.
\end{abstract}

Copyright (c) 2018 Institute of Advanced Engineering and Science. All rights reserved.

\section{Corresponding Author:}

Rekha N,

Department of Electronics \& Communication Engineering,

K.S. Institute of Technology,

Bangalore, India.

Email: rekhaphd2014@gmail.com

\section{INTRODUCTION}

The usage of the term 'noise' is closely associated with the surfacing of the unnecessary signals that potentially causes the degradation of the quality of the original signal [1], [2]. Any conventional electrical and electronic circuits is reported to have experience of 6 types of noises e.g. (a) Shot noise, (b) Burst noise (c) Thermal noise, (d) Flicker noise, (e) TDMA noise and (f) Avalanche noise [3], [4]. There are certain forms of the noise that are quite inevitable as they are originated from the original variation in the gauged quantity. Such forms of noises can be only mitigated by narrowing of the bandwidth as well as averaging of the signal [5] whereas there are also other forms of noises that are feasible to be minimized using different categories of techniques or by rectifying the defective configuration of wiring [6]. Example of such noises is radio frequency. There is certain category of noises that gives rise to potential amplification of the signal and usage of amplifier with minimal noise is selected for effectiveness. Although, it is significant to retain highest effectiveness of noise reduction techniques but there is something more important than this, which is to ensure that the considered system is majorly independent from the significant level of interference along with retention of minimal level of amplifier noise [7]. There are significant levels of problems particularly in the GSM signal where the customers experience various forms of noises while on board of any fast moving terrestrial vehicles. One of the typical problems in using the GSM-based network as well as GSM-based mobile devices is that there is always an interference as well as noise that are introduced over the signal of microphone [8]. This process only happens when the user mobile device is in transmitting phase along with its mobility stage. Although, such phenomenon is quite normal during the communication system in GSM 
but in reality there is an introduction of large number of noise as well as interference consisting of frequencies of fundamental original and its associated harmonics. According to the standards of GSM communication system, the rate of fundamental switching is considered as $217 \mathrm{~Hz}$ in approximate [9]. There are also particular segment of noise that is generated from the crystal and is highly periodic in nature. Formation of such noises only takes place when the signal generated from the microphone is correlated with the sinusoids that posse's similar crystal generated frequency. This is considered as potential level of noise. This problem is very critical as GSM channel doesn't only float acoustic in its network but it also floats different form of data. In existing time, with the presence of $3 \mathrm{G}$ and $4 \mathrm{G}$ based services, the customers chooses to render voice as secondary preference in comparison to different forms of data. There are various mobile apps running on the smartphone sustained by GSM-based services and network in which accuracy of data transmission is a demand of the user. Apart from this, the usage of telemedicine also adopts GSM based channel for transmitting clinical data. Hence, presence of any form of noises will result in the inaccurate diagnosis. Therefore, noises do have harmful effect in existing system. The existing filters can only perform cancellation of noises if the apriori information of the noise type is provided to the filter device. Therefore, we present a simple technique where such forms of unidentified noise can be detected and kept segregated to allow the seamless data transmission in the GSM-based channel. The proposed system also investigates the effect of the allocated power over the noise reduction by harnessing the potential of probability theory and analytical research methodology. Section 1.1 discusses about the existing literatures where different techniques are discussed for detection schemes of noises in file transmission lines followed by discussion of research problems in Section 1.2 and proposed solution in 1.3. Section 2 discusses about algorithm implementation followed by discussion of result analysis in Section 3. Finally, the conclusive remarks are provided in Section 4.

\subsection{Background}

This section discusses about the existing techniques towards controlling of the significant level of noises present during transmission of data through a wireless medium. This work is an extension to our prior review work [10]. Alias and Socoro [11] has presented a discussion of anomalous noise that arises from acoustic sensory network. Mostafa [12] have focused on addressing impulsive noise focusing on the conventional OFDM signals using blanking non-linearity. Zhang et al. [13] have introduced a methodology for minimizing noise by developing a selection method of microphone subsets that is directly linked with the lower cost of transmission. Antonanzas et al. [14] have presented a controller design with an aid of distributed least mean square algorithm. Duda [15] have investigated on noise reduction policy for the cellular phones that arises from road traffic condition. Ferras et al. [16] have designed a noise-resistant system that could recognize the acoustics in spite of the presence of noises over the medium. Liu et al. [17] have presented an optimization scheme for enhancing the scheduling schemes over wireless network by controlling different forms of the noise present in the wireless. Study towards interference cancellation was carried out by Ruder et al. [18] considering GSM by addressing the problems associated with the conventional keying process. Fathallah et al. [19] have presented discussion about noise minimization on optical network by introducing a modeling for identification of different forms of simple Gaussian noises. Study towards cancellation of clutter noise over GSM signal is carried out by Demissie [20]. Dixit and Nagaria [21] have used neural network for the purpose of noise minimization using least mean square approach.Nanda et al. [22] have investigated on the design of phased locked loop to be used in GSM applications for assisting in lowering the noises. Chen et al. [23] have used radial basis function for controlling noises of adaptive nature that further assists in an efficient recovery of the signal. Kim and Kim [24] have presented a technique for minimizing the bumblebee noise on GSM-based devices using hardware filters. Salmasi et al. [25] have used neural network for controlling minimization of active noises. Study towards minimizing flickering noise is carried out by Jiang et al. [26] that also in parallel assists in offering better input with enhanced linearity. Goosens et al. [27] have designed a unique filter for performing denoising of different forms of conventional noises. Ruippo et al. [28] have presented a study that emphasize on developing an oscillator that is controlled by voltage with highly less amount of phase noises operating at critical frequencies of GSM signals. Neeraja and Rao [29] have presented the comparative research for CDMA and GSM cellular networks over Handoff algorithm and the research is found helpful in improving cellular service. In Xie et al. [30] explained the data transmission mechanism based on the measurement acquired from railway based GSM, i.e., GSM-R and concluded that this work could be considered for theoretical researches. Tahcfulloh and Riskayadi [31] introduced the suitable propagation model to predict the path loss in GSM900 and significantly implemented over it. Therefore, it can be seen that there are various forms of the implementation techniques that directly or indirectly has attempted to perform noise reduction in the wireless channel. Not all the channels are from the GSM, but there are other form of mobile networks that bears similar form of noises just like that happens in GSM. All the associated research work 
has advantages in its own way as well as it is also shrouded with different forms of limitations too. The next section discusses about the limitation of the existing techniques that the proposed system chooses to address for overcoming the problems of noise cancellation in GSM channel.

\subsection{Research Problem}

The significant research problems are as follows:

a. Existing techniques are capable of identifying only some standard noises generated from static sources and is completely incapable of identification of noises for any fast moving sources.

b. The frequently used case study for multimedia files is acoustic in noise cancellation in GSM whereas GSM also forwards images, text, executables, etc. that were never considered.

c. Till date, there are few studies that have correctly represented the level of noise arisen from GSM-based device before applying it to any noise cancellation technique.

d. Impact of allocated power on the GSM device towards the noises being generated has never been investigated in the existing system.

Therefore, the problem statement of the proposed study can be stated as "It is highly computationally challenging task to perform a precise identification of undefined forms of noises influenced by the allocated power in the GSM signals for an effective noise cancellation."

\subsection{Proposed Solution}

This proposed solution is an extension of our prior stochastic model towards GSM noise cancellation.We emphasizes that suppressing the noise can be effectively carried out if the identification is carried out in a precise manner. Therefore, in the proposed solution, we emphasize on applying probability theory where identification of noise by power allocation is carried out. The schematic diagram is shown in Figure 1.

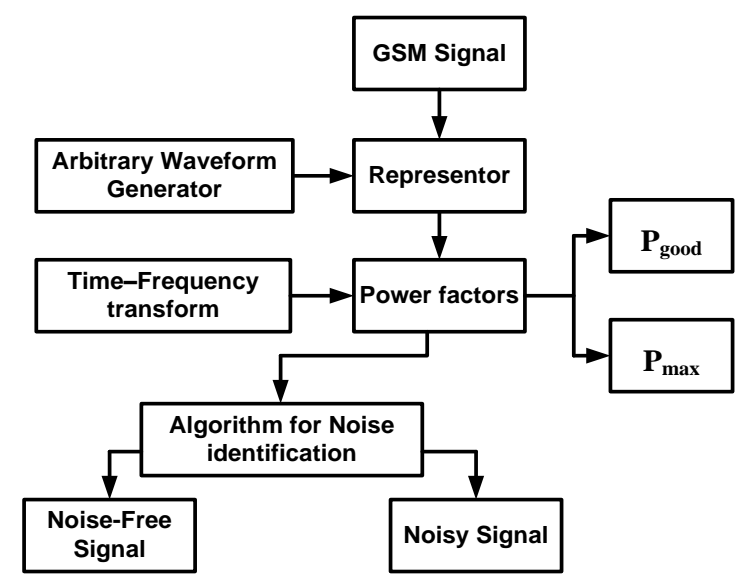

Figure 1 Schema of Proposed Methodology

The proposed system considers an analytical research methodology where it is considered that a GSM signal generated from the fast-moving vehicle is always incorporated with undefined noises along with the significant level of interference. Therefore, the proposed system introduced a mechanism where such undefined noise is represented by the GSM signal as well as from arbitrary waveform generator to perform modeling on the undefined form of noises in GSM signal. We then develop a simple mechanism of power factors where the power of good signals as well as the maximum power required is computed. An algorithm for identification of noise is constructed that after processing segregates good from the bad signal in GSM communication system. The contribution of the proposed system is its simplistic design with faster response time to perform identification of good signals for the vehicles moving at a faster velocity. The next section discusses the algorithm being implemented for this purpose.

\section{ALGORITHM IMPLEMENTATION}

The primary basis of the proposed algorithm is to perform a precise identification of all form of radio-frequency noises present in the acoustic GSM signal. The basic idea behind the proposed study is to 
precisely represent a space where the identification of the GSM signals is required to be carried out. Such exploration of the space is always indexed with the aid of certain attributes from the surveilled GSM signal with the help of a new variable called as represented. Although, such identification of different forms of GSM signals could be effectively carried out using discriminative, stochastic, neuronal, approaches, but proposed system completely uses a probabilistic approach to do the same. The new term represents an amount that is estimated from the GSM signals that are aggregated from the transmitter that is a collection of all the attributes influencing the communication quality. An empirical form of the represent can be obtained by computing level of power of the constructive signal $\left(\mathrm{P}_{\text {good }}\right)$ divided by that of interference of radiofrequency $\left(\mathrm{P}_{\text {noise }}\right)$ obtained from the GSM signal as follows:

$$
R(t)=\frac{P_{\text {good }}}{P_{\text {noise }}}
$$

However, the research challenge is to distinguish the form of noisy signals of GSM concerning their extent of power being introduced (or allocated) on a specific GSM channel. We solve this problem by harnessing the characteristics of the broadband belong to this form of transient GSM signal. Therefore, the study considers a fixed band of frequency for GSM that ranges from $921 \mathrm{MHz}$ as minimum frequency and $925 \mathrm{MHz}$ as maximum frequency. Hence, there is no significant difference between $\mathrm{P}_{\text {good }}$ and $\mathrm{P}_{\text {noise }}$ much. Therefore, it is feasible for approximating the noises arising from radio-frequency interference by considering the greatest feasible extent of power $\left(\mathrm{P}_{\text {noise }}\right)$ keeping the similar range of bands of frequency $\left(\mathrm{P}_{\max }\right)$.

$$
R(t)=\frac{P_{\text {good }}}{P_{\max }}
$$

The proposed system initially computes the transform of time-frequency followed by utilizing the coefficients for estimating the represent. The calculation of the representor is carried out by assessing both the time and frequency pattern followed by $\mathrm{P}_{\text {good }}$ and $\mathrm{P}_{\max }$. This computation is carried out at a regular time interval of $t$. We consider that if there is any presence of transient factor during $t$ time than all the GSM signals are subjected to mixing so that it is challenging to distinguish the signal by their power attributes. Therefore, the system considers $(t-1)$ time duration allocated for $\mathrm{P}_{\text {good }}$ such that $\mathrm{P}_{\text {good }}(t)$ is equivalent to $\mathrm{P}_{\text {good }}$ $(t-1)$. The computation of $\mathrm{P}_{\max }$ is carried out by considering the highest value on the band of frequency equivalent to a standard form of GSM signal. Similarly, the proportion between the extents of power provides an empirical value of represented $R$ at $t$ time followed by repetition of the similar method over all the duration of time correlating to time and frequency form of data. From conventional GSM signal, the rate of the symbol is found to be minimum of 270 kilobits per second while the duration of time and frequency pattern is found to be 1 millisecond. The algorithm is constructed by associating the descriptor with the error rate value where it is required to operate at 3.7 microseconds on the scale of transmission bit for noisy GSM signal. The study has aggregated the estimation of the proportion of $\mathrm{P}_{\text {good }} / \mathrm{P}_{\text {noise }}$ on multiple windows that successfully overlap with each other. The significant steps of the algorithm are as follows:

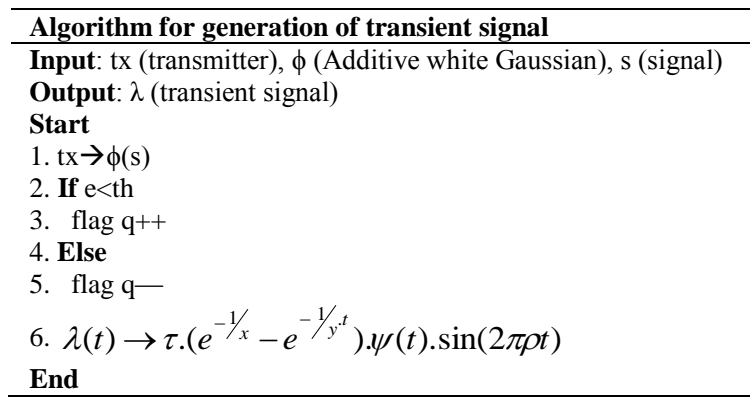

After the pattern of the representor is extracted, we also extract numerous second-order attributes. The preliminary parameter represents a minimum that depicts occurrences of various transients in the GSM signal while the second attribute depicts the corrupted level of the signal to noise ratio during the occurrence of the transient. It is to be noted that such forms of occurrences associated with the transient have a deeper impact on transmitting GSM signal. The estimation of the minima is carried out by using cut-off value where a minimum represents all the numerical value that is lower than this cut-off value. The significance of this cut- 
off value $t h$ is very essential as the cut-off value much lower than it could result in missing of certain minima whereas if the numerical value of cut-off is much higher than it could result in incorporating falsified detection. Therefore, there is always a generation of errors within the summation of all the minima values. The proposed system considers assessing the GSM signal that is characterized by 2.4 million points along with the frequency of sampling corresponding to $2.4 \mathrm{GHz}$. This is an evident depiction of the window of 1 milliseconds time duration. The proposed algorithm initially assesses the GSM signal evolution over an increased duration of time considering an increasing number of points. The algorithm than focuses on capturing the number of transient signals that is characterized by the shortest duration owing to the implication of frequency of finer sampling origin. The study considers the shortest frequency to be 20 nano seconds. The process of capturing numerical value is performed by multiple steps of operation. The first step is to establish the communication system between the mobile devices (i.e., transmitter) so that the level of GSM signal is configured properly. The algorithm also uses additive Gaussian noise to incorporate transient noise in radio-frequencies of GSM signal followed by estimating all the error rates value as well as identification of class to index the extracted signal. The assessment of the noise identification is carried out for more than the duration of 1 millisecond. The next step is to write a conditional statement for both the category of noises to perform better indexing of the measurement signals of the radio-frequencies associated with the transient noises that are found to have a significant impact on the GSM signal quality. The first conditional statement suggests that if the error rate is found less than the cut-off percentage than it is considered to be the better signal quality of GSM signal whereas if the error rate is found more than the cutoff percentage than it is considered to be the inferior quality of GSM signal. The proposed study considers the error rate as the rate of error that has been evolved up before any other form of correction of error. After the minima attribute as well as a summation of the second minima attribute is extracted from single assessment, all these values are finally evaluated to check its preciseness. These results in storage of such numerical values temporarily that will further be acted as orientation based. The segregation of the GSM signal is carried out in two ways where the first classifier recons to superior signal quality while other for inferior signal quality. The proposed system empirically expresses a recursive transient signal as follows,

$$
\lambda(t) \rightarrow \tau \cdot\left(e^{-1 / x}-e^{-1 / y^{t}}\right) \cdot \psi(t) \cdot \sin (2 \pi \rho t)
$$

In the above expression, where $\lambda$ represents amplitude, $x$ represents time duration, $y$ represents rinse time, $\psi$ represents unit step function, and $\rho$ represents frequency. The validation of the proposed algorithm was carried out by implying it to categorize the monitored GSM signals that are developed using a hypothetical form of transient signals. The study considers that there are two different form of transient GSM signal that is captured from the vehicle that is moving fast. The algorithm considers a similar variation of the interval of time as that of orientation base GSM signal as well as similar computation is also applicable to the fluctuation of the signal to noise ratio. The algorithm implementation scenario is as shown in Figure 2.

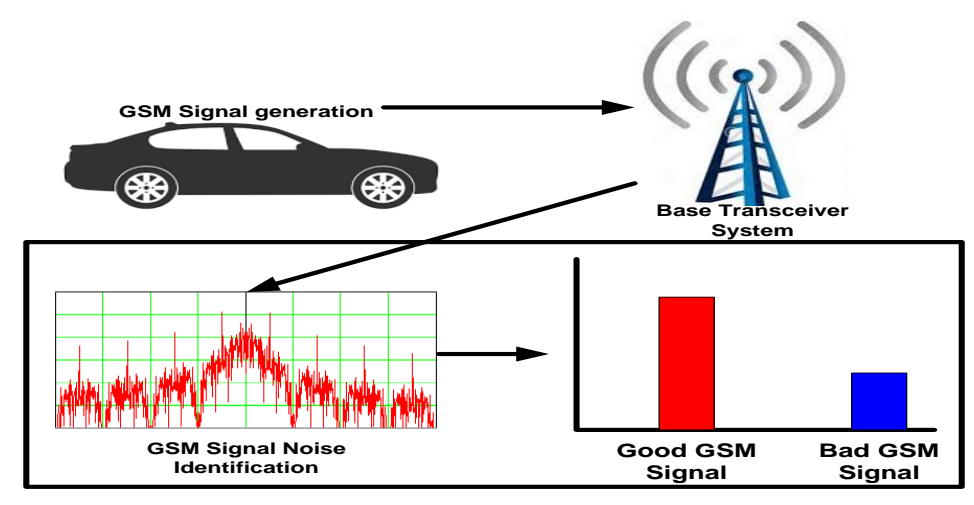

Figure 2. Algorithm Implementation Scenarios

\section{RESULT ANALYSIS}

The implementation of the proposed study is carried out in Matlab where once the test values are done with the preliminary round of monitoring, the orientation base of the GSM signal is found to consist of the maximum good signal as well as very less bad signals. Such forms of the numerical scenarios assist us to 
encapsulate the complete GSM signal inflicted by noise. It also assists in precisely defining the spectrum between the classes of the two forms of signals with special significance to the spectrum points that are very close to the peak values of the corresponding GSM signals with a different form of random noises mixed with additive white Gaussian noise. Figure 3 show the signal analysis of proposed system.
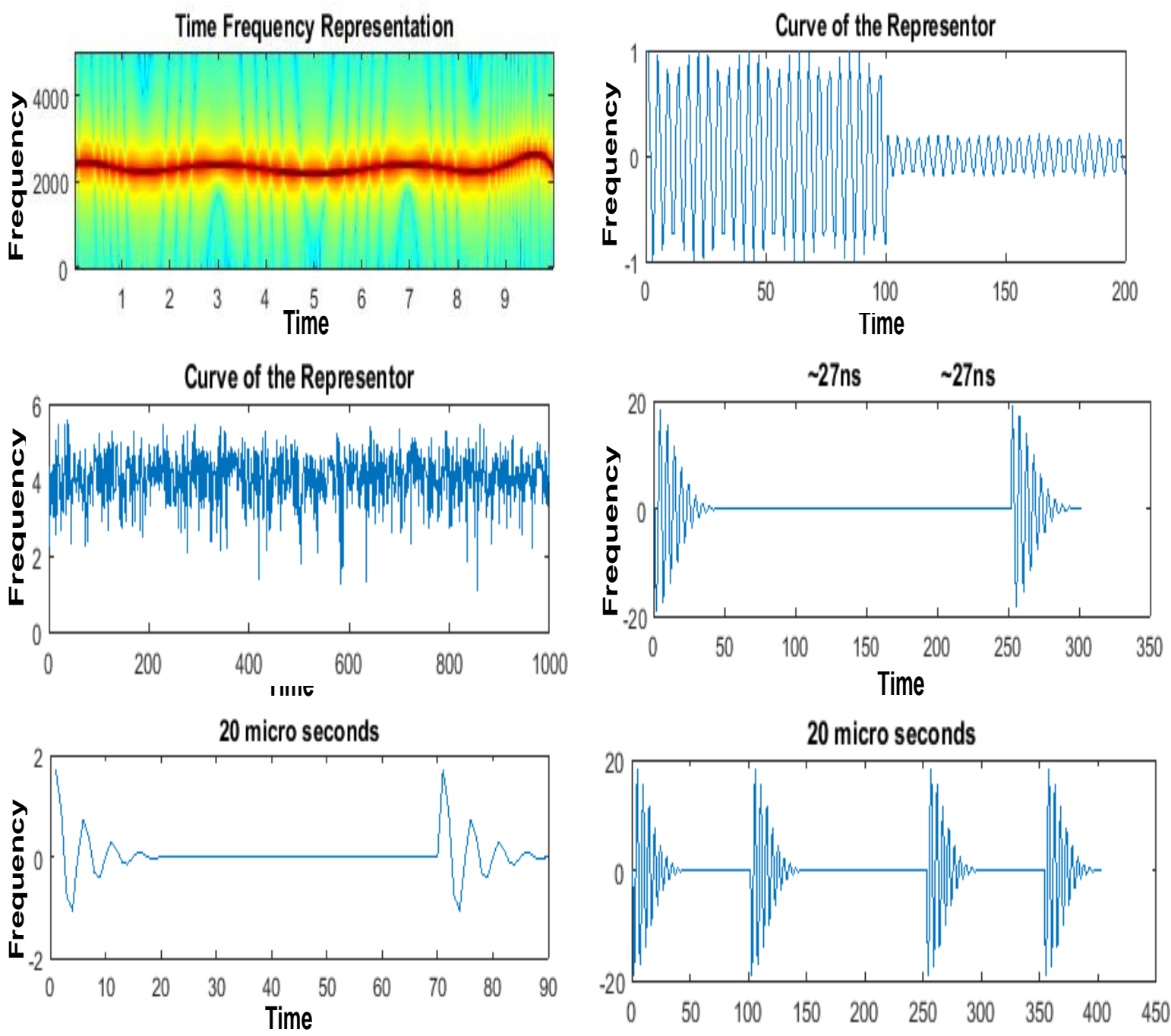

Figure 3. Signal Analysis of Proposed System

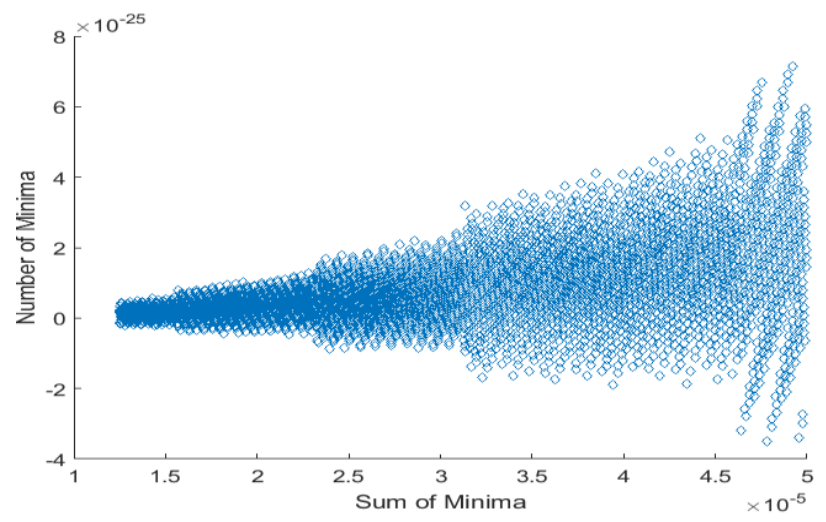

Figure 4. Scatter Plot for Noise Identification 
Figure 4 outlines the scatter plot of the noise identification where it can be seen that dense area of scattering represents the greater extent of noise whereas scare area represents the lower extent of transient noise. The overall algorithm processing time is 0.1662 seconds and is found to offer cost-effective solution towards the noise detection in GSM signal.

\section{CONCLUSION}

This proposed paper has presented a mechanism where an identification of undefined transient noises arising from the radio-frequencies of the GSM signal is investigated. The prime objective of the presented study is to define a tool that can be installed in any terrestrial moving vehicles for performing identification of the transmission quality of the GSM signal. Using analytical methodology, the proposed system considers a typical relationship between allocated powers as well as the level of undefined noises obtained by a mix of additive white Gaussian noise and random noise. The outcomes show that proposed system is capable of performing comprehensive classification of different forms of noises being generated by the GSM Signal.

\section{REFERENCES}

[1] B. Munegowda, "Denoising Audio Signal from Various Realistic Noise using Wavelet Transform," GRIN Verlag, 2016.

[2] N. Silver, "The Signal and the Noise: The Art and Science of Prediction," Penguin UK, 2012.

[3] R. C. Li, "RF Circuit Design," John Wiley \& Sons, 2012.

[4] B. M. Wilamowski, J. D. Irwin, "Fundamentals of Industrial Electronics," CRC Press, 2016.

[5] R. W. Middlestead, "Digital Communications with Emphasis on Data Modems: Theory, Analysis, Design, Simulation, Testing, and Applications," John Wiley \& Sons, 2017.

[6] R. C. Mullin, Phil Simmons, "Electrical Wiring Residential," Cengage Learning, 2017.

[7] A. Kay, "Operational Amplifier Noise: Techniques and Tips for Analyzing and Reducing Noise," Elsevier, 2012.

[8] S. Misra, S. Goswami, "Network Routing: Fundamentals, Applications, and Emerging Technologies," John Wiley \& Sons, 2017.

[9] M. Sauter, "From GSM to LTE: An Introduction to Mobile Networks and Mobile Broadband," John Wiley \& Sons, 2010.

[10] Rekha N and F. Jabeen. "Article: Study on Approaches of Noise Cancellation in GSM Communication Channel," Communications on Applied Electronics 3(5):5-11, Published by Foundation of Computer Science (FCS), NY, and USA, 2015.

[11] F. Alías and J. C. Socoro, "Description of Anomalous Noise Events for Reliable Dynamic Traffic Noise Mapping in Real-Life Urban and Suburban Soundscapes," Applied Science Journal, vol.7, no.146, 2017.

[12] M. Mostafa, "Stability Proof of Iterative Interference Cancellation for OFDM Signals With Blanking Nonlinearity in Impulsive Noise Channels," in IEEE Signal Processing Letters, vol. 24, no. 2, pp. 201-205, Feb. 2017.

[13] Z. Jie, S.P.Chepuri, R.C. Hendriks, and R.Heusdens, "Microphone Subset Selection for MVDR Beamformer Based Noise Reduction," IEEE/ACM Transactions on acoustics, speech and language processing, 2017.

[14] C. Antoñanzas, M. Ferrer, M. Diego and A. Gonzalez, "Blockwise Frequency Domain Active Noise Controller over Distributed Networks," Applied Science Journal, vol.6, 2016.

[15] P. Duda, "Processing and Unification of Environmental Noise Data from Road Traffic with Spatial Dimension Collected through Mobile Phones," Journal of Geoscience and Environment Protection, 2016, 4, 1-26.

[16] M. Ferràs, S. Madikeri, P. Motlicek, S. Dey and H. Bourlard, "A Large-Scale Open-Source Acoustic Simulator for Speaker Recognition," in IEEE Signal Processing Letters, vol. 23, no. 4, pp. 527-531, April 2016.

[17] J. S. Liu, C. H. R. Lin, and J. Tsai, "Delay and Energy Tradeoff in Energy Harvesting Multi-Hop Wireless Networks With Inter-Session Network Coding and Successive Interference Cancellation," in IEEE Access, vol. 5, pp. 544-564, 2017.

[18] M. A. Ruder, A. M. Lehmann, R. Schober, and W. H. Gerstacker, "Single Antenna Interference Cancellation for GSM/VAMOS/EDGE Using \$L_\{p\}\$-Norm Detection and Decoding," in IEEE Transactions on Wireless Communications, vol. 14, no. 5, pp. 2413-2425, May 2015.

[19] H. Fathallah, A. Bentrcia, H. Seleem, "Efficient interference cancellation detector for asynchronous upstream optical code division multiple access-passive optical networks with mixed Poisson-Gaussian noise," IEEE- Published in IET Communications, 2014.

[20] B. Demissie, "Clutter cancellation in passive radar using GSM broadcast channels," IEEE- Published in IET Radar, Sonar, and Navigation, 2014.

[21] S. Dixit and D. Nagaria, "Neural network implementation of Least-Mean-Square adaptive noise cancellation," 2014 International Conference on Issues and Challenges in Intelligent Computing Techniques (ICICT), pp. 134-139, 2014.

[22] U. Nanda, D. P. Acharya and S. K. Patra, "Design of a low noise PLL for GSM application," International Conference on Circuits, Controls and Communications (CCUBE), Bengaluru, pp. 1-4, 2013.

[23] H. Chen, Y. Gong and X. Hong, "Adaptive noise cancellation with fast tunable RBF network," Sensor Signal Processing for Defence (SSPD 2012), London, pp. 1-5, 2012 
[24] T. S. Kim and S. W. Kim, "A method for reducing a bumblebee noise generated by a GSM technology in a smartphone," 12th International Conference on Intelligent Systems Design and Applications (ISDA), Kochi, pp. 927-930, 2012.

[25] M. Salmasi, H. Mahdavi-Nasab and H. Pourghassem, "Comparison of Feed-Forward and Recurrent Neural Networks in Active Cancellation of Sound Noise," International Conference on Multimedia and Signal Processing, Guilin, China, pp. 25-29, 2011.

[26] P.C. Jiang, T.T. Yan, J. Jin, and J.J. Zhou, "A Low Flicker Noise and High IIP2 Downconversion Mixer for Zero-IF GSM Receiver", IEEE, 2011.

[27] G. Bart, H. Luong, A. Pizurica, and W. Philips, "An Improved Non-Local Denoising Algorithm," Research gate, 2008.

[28] P. Ruippo, T. A. Lehtonen and N. T. Tchamov, "An UMTS and GSM Low Phase Noise Inductively Tuned LC VCO," in IEEE Microwave and Wireless Components Letters, vol. 20, no. 3, pp. 163-165, March 2010.

[29] S. Neeraja and G. S. Rao "A Comparative study on Handoff Algorithms for GSM and CDMA Cellular Networks," International Journal of Electrical and Computer Engineering (IJECE), vol. 7(3), 2017.

[30] T. Xie, "Analysis of Data Transmission Method based on GSM-R Network and Teaching Platform For Wireless Network", Indonesian Journal of Electrical Engineering and Computer Science, vol. 11(7), pp.3590-3596, 2013.

[31] S. Tahcfulloh and E. Riskayadi, "Optimized Suitable Propagation Model for GSM 900 Path Loss Prediction", Indonesian Journal of Electrical Engineering and Computer Science, vol. 14(1), pp. 154-162, April 2015.

\section{BIOGRAPHIES OF AUTHORS}
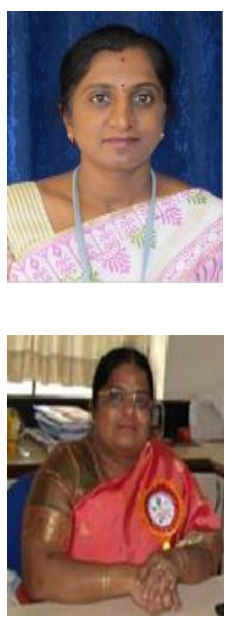

Rekha $\mathbf{N}$ is Associate Professor in Department of Telecommunication Engineering, K. S. Institute of Technology, Bengaluru, and Karnataka, India. She received B.E degree in Electronics and Communication Engineering from Visvesvaraya Technological University, Belagavi, Karnataka in the year 2002 and M.Tech degree in Digital Electronics, from the same university in the year 2009. She is currently pursuing her Ph.D. degree in the Department of Electronics and Communication Engineering, Visvesvaraya Technological University, Belagavi. Her research interests include wireless communication; secure communication networks, Data Communication, MATLAB modeling, and simulation.

Dr. Fathima Jabeen, Ph.D., is Principal of Islamiah Institute of Technology, Bengaluru, and Karnataka, India. She is a Supervisor for the Ph.D. scholars at Visvesvaraya Technological University, Belagavi, Dr. MGR University, Jain University and also an external examiner for Thesis evaluation/ Public Viva-voce of Ph.D. students. She has been in the teaching for profession courses under UG/PG level for nearly 29 years. She is a reviewer for several National and International journals. Dr. Fathima Jabeen is a Fellow Member of the Institution of Engineers (INDIA). She is also a life member of several professional bodies, including Indian Society for Technical Education (ISTE) and member IEEE.She has nearly 35 publications under her name. Her area of interest includes Communication, Instrumentation, Embedded Systems, Wireless communication, Automotive Electronics, and VLSI. 